\title{
Nitric oxide Does Not Trigger Early Programmed Cell Death Events but May Contribute to Cell-to-Cell Signaling Governing Progression of the Arabidopsis Hypersensitive Response
}

\author{
Chu Zhang, ${ }^{1}$ Kirk J. Czymmek, ${ }^{2}$ and Allan D. Shapiro, ${ }^{1,2}$ \\ ${ }^{1}$ Department of Plant and Soil Sciences, Delaware Agricultural Experiment Station, College of Agriculture and Natural \\ Resources, University of Delaware; ${ }^{2}$ Department of Biological Sciences, University of Delaware, Newark, U.S.A.
}

Submitted 28 April 2003. Accepted 2 July 2003.

\begin{abstract}
Nitric oxide (NO) has been suggested to play a role in the hypersensitive response (HR). Single- and double-label fluorescence microscopy experiments were conducted using Arabidopsis leaves infected with Pseudomonas syringae pv. tomato DC3000 carrying either avrB or avrRpt2. Kinetics of NO production were followed by measurement of green 4amino-5-methylamino-2', $7^{\prime}$-difluorofluorescein (DAF-FM) triazole fluorescence in leaves coinfiltrated with DAF-FM diacetate. Kinetics of hypersensitive cell death were followed by measurement of cytoplasmic red fluorescence following internalization of coinfiltrated propidium iodide through compromised plasma membranes. Neither NO accumulation nor cell death was seen until approximately 3 h postinoculation of Columbia leaves with DC3000 avrB or approximately $5.5 \mathrm{~h}$ post-inoculation with DC3000•avrRpt2. Subsequent NO accumulation kinetics closely paralleled HR progression in both Columbia and ndr1-1 mutant plants. These data established that NO accumulation does not happen sufficiently early for NO to be a signaling component controlling HR triggering. NO accumulation did contribute to the $H R$, as proven by an approximately 1-h delay in cell death kinetics caused by an NO scavenger or an NO synthase inhibitor. NO was first seen as punctate foci at the cell surface. Subsequent NO accumulation patterns were consistent with NO being an intercellular signal that functions in cell-to-cell spread of the HR.
\end{abstract}

Additional keywords: disease resistance, signal transduction.

The plant hypersensitive response (HR) to high titers of avirulent pathogens is assayed macroscopically by the visible collapse of leaf tissue. It is caused by pathogen-elicited programmed cell death (PCD) in most cells of the infected leaf. Loss of ions through compromised plasma membranes is followed by efflux of water. Transpirational loss of water leads to leaf desiccation and collapse. The HR is correlated with genefor-gene disease resistance, and hypersensitive cell death is thought to help contain pathogen spread (Goodman and Novacky 1994).

Nitric oxide (NO) accumulation has been implicated in the HR. Arabidopsis leaf collapse elicited by Pseudomonas syringae bacteria carrying the avirulence (avr) gene avrRpml was blocked by inhibitors of mammalian nitric oxide syn-

Corresponding author: Allan D. Shapiro, E-mail: ashapiro@udel.edu. thase (NOS) (Delledonne et al. 1998). The same study showed modest (approximately fourfold) enhancement in bacterial growth in plants coinfiltrated with the NOS inhibitors. This study further supported a role of NO in the HR by showing that NOS inhibitors as well as an NO scavenger could partially block cell death elicited in soybean cell cultures by avirulent $P$. syringae. An NOS inhibitor also delayed the HR shown by tobacco leaves to avirulent Ralstonia solanacearum (Huang and Knopp 1998). Similar experiments have implicated NO as a signal leading to hypersensitive cell death in Arabidopsis cell cultures responding to avirulent $P$. syringae (Clarke et al. 2000), in rice cell cultures responding to a Magnaporthe grisea-derived race-specific elicitor of plant PCD (Hu et al. 2003), and in tobacco epidermal peels responding to a non-race-specific, Phytophthora cryptogeaderived elicitor (cryptogein) (Foissner et al. 2000). NO donors have been shown to induce PCD of cell cultures when added either alone or in combination with exogenous $\mathrm{H}_{2} \mathrm{O}_{2}$ (Clarke et al. 2000; de Pinto et al. 2002; Delledonne et al. 1998, 2001; Garcês et al. 2001; Hu et al. 2003; Pedroso et al. 2000a,b; Zottini et al. 2002). However, NO donors also have been shown to be cytoprotective under other conditions (Beligni et al. 2002; Beligni and Lamattina 1999; Delledonne et al. 2001; Huang et al. 2002b). Consistent with a role for NO in defense responses, NOS-like activity was shown to be induced in tobacco plants infected with avirulent Tobacco mosaic virus (TMV) (Durner et al. 1998) or R. solanacearum (Huang and Knopp 1998). This NOS-like activity recently has been purified from TMV-infected tobacco leaves (Chandok et al. 2003). A protein homologous to the $\mathrm{P}$ component of the glycine decarboxylase complex was shown to be responsible for this activity.

Although these studies have established that NO does play a role in the HR, the precise function of NO was not established. Knowing the subcellular locations in which NO accumulates and the kinetics of accumulation would help address this issue. Although these questions have never been addressed in intact plants responding to avirulent pathogens, real-time imaging was used to follow NO accumulation in tobacco responding to cryptogein (Foissner et al. 2000). Three minutes after addition of cryptogein to tobacco epidermal peels, NO was seen to accumulate in chloroplasts. Three minutes later, NO accumulation also was noted in the nucleus, cytoplasm, peroxisomes, and along the plasma membrane. Consistent with these results, other studies have localized basal NOS enzyme activity to peroxisomes (Barroso et al. 1999) or cytosol (Delledonne et al. 
1998; Ribeiro et al. 1999). Immunoreactivity in uninfected plants with antibodies raised against mammalian NOS enzyme showed staining in the peroxisomes and chloroplasts (Barroso et al. 1999) or in the cytoplasm and nucleus (Ribeiro et al. 1999).

The dye used to localize NO accumulation in vivo, 4,5-diaminofluorescein diacetate (DAF2-DA), will only report NO levels under neutral or basic $\mathrm{pH}$ conditions (Kojima et al. 1999). The triazole product of interaction with NO does not show appreciable fluorescence under the $\mathrm{pH}$ condition expected in acidic plant compartments such as the apoplast. As such, NO accumulation either extracellularly or in an internal acidic compartment would have been missed by DAF2-DAbased studies. Indeed, extracellular NO was detected in soybean and Arabidopsis cell cultures inoculated with avirulent Pseudomonas syringae bacteria using an assay based on NOmediated conversion of exogenously supplied hemoglobin to methemoglobin (Clarke et al. 2000; Delledonne et al. 1998).

In this work, we employed a different dye, 4-amino-5-methylamino-2',7'-difluorofluorescein (DAF-FM) diacetate, to report the kinetics of accumulation of NO during the HR. The chemistry of interaction with NO is similar to that of DAF2DA with improved dye photostability and sensitivity for NO detection. Importantly, the triazole form of this dye (the NO adduct) fluoresces strongly not only at neutral or basic $\mathrm{pH}$ but also at the acidic $\mathrm{pH}$ found in the apoplast (Itoh et al. 2000; Kojima et al. 1999). DAF-FM diacetate was employed in double label fluorescence experiments that also followed internalization of propidium iodide (PI) as a marker of hypersensitive cell death. Contrary to expectations based on results with cell cultures, NO accumulation was not an early, intracellular signaling event in the HR. Instead, NO accumulation was first seen at the cell surface very close to the time of hypersensitive cell death. NO appeared to function in cell-to-cell signaling governing HR progression.

\section{RESULTS}

Arabidopsis leaves show NOS-dependent NO accumulation in response to infection with avirulent bacteria.

DAF-FM diacetate is cell-permeant. In plants, it can be deacetylated by either extracellular or intracellular esterases. DAF-FM then reacts with NO to make a covalent triazole compound (DAF-FM triazole) with 160-fold increased fluorescence quantum efficiency (Kojima et al. 1999). This bright green fluorescence can be captured by epifluorescence microscopy (Itoh et al. 2000). All figure panels in this article represent typical results from observations of at least triplicate leaves for each experiment, with every experiment repeated at least three times.

The specificity of this method of NO detection in intact Arabidopsis leaves first was confirmed. Leaves were infiltrated with a $5 \mathrm{mM}$ solution of the $\mathrm{NO}$ donor sodium nitroprusside (SNP) and subsequently infiltrated with DAF-FM diacetate through the same needle holes. Infiltration with DAF-FM diacetate was 15 min prior to detachment of the leaf from the plant for microscopy with this and all subsequent experiments. In images taken $4 \mathrm{~h}$ postinfiltration with SNP, fluorescence was seen throughout the leaf (Fig. 1A). Consistent with results from Arabidopsis cell cultures (Clarke et al. 2000), this level of SNP induced cell death in Arabidopsis leaves (seen as macroscopic leaf collapse) at later timepoints (data not shown). Coinfiltration of the NO scavenger 2-(4-carboxyphenyl)-4,4,5,5tetramethylimidazoline-1-oxyl-3-oxide potassium salt (cPTIO) with SNP eliminated the DAF-FM triazole signal (Fig. 1B). Mock inoculation of leaves with $10 \mathrm{mM} \mathrm{MgCl}_{2}$ did not lead to appreciable DAF-FM triazole fluorescence signal (Fig. 1C).
By contrast, inoculation with Pseudomonas syringae pv. tomato DC3000 (hereafter called DC3000) carrying a plasmidborne copy of $a v r B$ at $2 \times 10^{7}$ bacteria per $\mathrm{ml}$ did lead to many cells showing bright fluorescence $4 \mathrm{~h}$ postinoculation (Fig. 1D). This bright fluorescence was eliminated if cPTIO was coinfiltrated with the bacteria (Fig. 1E).

Next, the biosynthetic origin of NO produced postinfection with avirulent bacteria was established. Consistent with a requirement for an NOS-like enzyme, coinfiltration of the inhibitor of mammalian NOS enzymes $\mathrm{L}_{-} \mathrm{N}^{\mathrm{G}}$-monomethyl-arginine monoacetate (L-NMMA) with the bacteria eliminated the fluorescence signal (Fig. 1F). Control experiments showed that neither L-NMMA nor cPTIO affected bacterial viability as assessed by colony formation on NYG (nitrogen source [peptone], yeast extract, and glycerol) agar plates (C. K. Worley, C. Zhang, and A. D. Shapiro, unpublished data). Nitrate reductase, rather than an NOS-like enzyme, has been implicated in abscisic acid-induced NO production in guard cells (Desikan et al. 2002). The nia1-1/nia2-5 double mutant shows greatly diminished nitrate reductase activity due to loss-of-function mutations in each of the two nitrate reductase genes (Wilkinson and Crawford 1993). Direct comparison of infected Columbia wild-type plants (Fig. 1G) with infected nial-1/nia2-5 doublemutant plants (Fig. 1H) from the same experiments showed no appreciable differences in NO accumulation at any timepoint examined (pictures shown from timepoint taken $4.5 \mathrm{~h}$ postinoculation). These results suggest that an NOS-like enzyme (Chandok et al. 2003), rather than nitrate reductase, is responsible for NO synthesis during the Arabidopsis HR.

\section{NO accumulation is first seen close to the time of the first hypersensitive cell death events and is not seen in response to virulent bacteria.}

The kinetics of NO accumulation were compared next to those of hypersensitive cell death. The first PCD events occur approximately $3 \mathrm{~h}$ postinoculation with DC3000.avrB as assessed by quantitative, conductance-based assays of ion leakage (Zhang et al., submitted). Double label experiments were performed to compare kinetics of NO accumulation and PCD in the same fields of cells. PCD was assessed by internalization of PI. PI will emit red fluorescence upon excitation. Binding of PI to RNA or DNA enhances the fluorescence quantum efficiency by 20- to 30-fold (Arndtjovin and Jovin 1989). PI does not cross intact cell membranes and thus can be used for identifying dead cells in a population (Koyama et al. 2001). DAF-FM triazole fluorescence is green; therefore, appropriate choice of filter sets allowed facile discrimination between the two labels. Chloroplast autofluorescence was distinguished from PI fluorescence based on differences in emission spectra.

The time course shown in Figure 2 was taken from a single day's experiment and should be considered typical results. DAF-FM triazole fluorescence and cytoplasmic PI fluorescence were both first seen in isolated cells approximately $3 \mathrm{~h}$ postinoculation. This time of onset of the responses varied slightly from day to day, the range being from $2 \mathrm{~h} 50 \mathrm{~min}$ to 3 h 20 min postinoculation. Similar variation was seen when cell death was measured with ion leakage-based assays (C. Zhang and A. D. Shapiro, unpublished data). However, the results prior to this timepoint were consistent in all trials of the experiment. Neither NO accumulation nor cell death was seen within approximately the first $3 \mathrm{~h}$ postinfection. In the experiment shown, the 3-h 15-min timepoint captured the highly transient state in which NO accumulation could be seen prior to PI internalization. Although DAF-FM triazole staining was faint, the higher magnification and enhanced contrast shown with the inset (Fig. 2C) allowed a clear difference to be seen when compared with the 2-h timepoint. Fluorescence appeared to be exclusively 

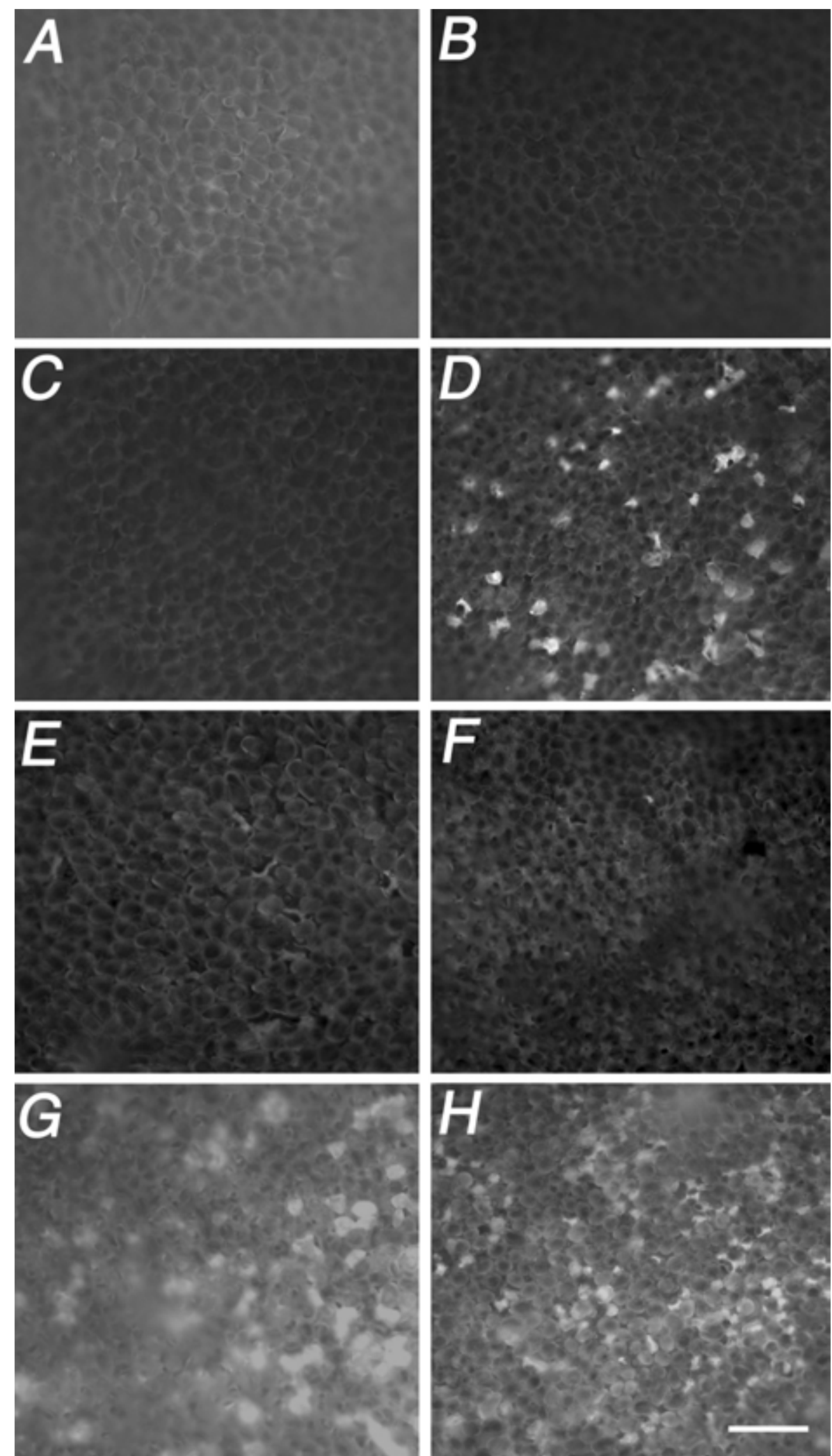

Fig. 1. Nitric oxide (NO) production in response to DC3000-avrB required nitric oxide synthase (NOS)-like activity. A, NO release from sodium nitroprusside (SNP) could be detected by fluorescence resulting from interaction with 4-amino-5-methylamino-2',7'-difluorofluorescein (DAF-FM). B, NO scavenger 2-(4-carboxyphenyl)-4,4,5,5-tetramethylimidazoline-1-oxyl-3-oxide potassium salt (cPTIO) prevented NO accumulation following release from SNP, confirming the specificity of the fluorescence signal as indicative of NO levels. C, Mock inoculation with $10 \mathrm{mM} \mathrm{MgCl}$ did not lead to $\mathrm{NO}$ accumulation ( $4 \mathrm{~h}$ postinoculation). D, Inoculation with DC3000 $\operatorname{avrB}$ at $2 \times 10^{7}$ bacteria $\mathrm{ml}^{-1}$ led to high level accumulation of NO (4 h postinoculation) E, The NO scavenger cPTIO blocked NO accumulation when co-infiltrated with DC3000.avrB (assessed 4 h postinoculation). F, The NOS inhibitor L- ${ }^{\mathrm{G}}$ monomethyl-arginine monoacetate blocked NO accumulation when co-infiltrated with DC3000-avrB (assessed 4 h postinoculation). No significant differences were seen in NO accumulation in response to infection with DC3000 avrB between $\mathbf{G}$, wild-type Columbia plants and $\mathbf{H}$, nial/nia2 double mutant plants. Pictures were taken $4.5 \mathrm{~h}$ postinoculation, and the plants shown were from the same experiment. Scale bar $=100 \mu \mathrm{m}$ (all panels at identical level of magnification) 


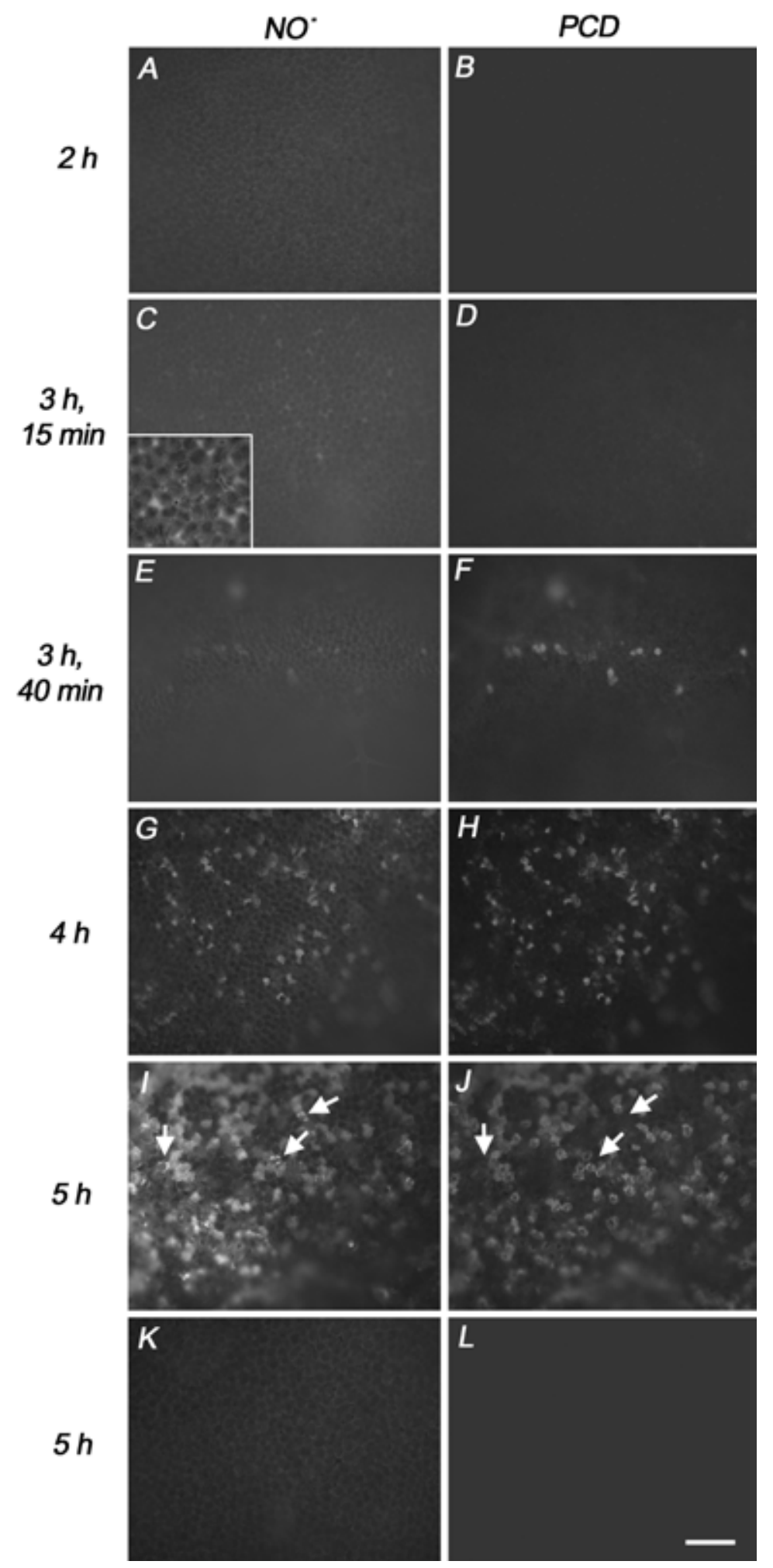

Fig. 2. Nitric oxide (NO) accumulation was a specific response to avirulent bacteria and was first seen immediately prior to the time of hypersensitive cell death. All pictures were taken from an experiment performed within a single day and represent typical results. Pairs of images at each indicated timepoint were from the same leaf areas. Times postinoculation with DC3000 avrB at $2 \times 10^{7}$ bacteria $\mathrm{ml}^{-1}$ are indicated for each image pair. 4-amino-5methylamino-2',7'-difluorofluorescein (DAF-FM) diacetate and propidium iodide (PI) were co-infiltrated 15 min prior to leaf harvest. A and B, No fluorescence was seen above background levels in either picture. C and D, In this experiment, the very transient state in which DAF-FM triazole fluorescence can be documented prior to PI internalization was captured. C, The inset shows a region with twofold greater magnification and enhancement of contrast. The faint DAF-FM triazole signal appeared to be exclusively extracellular. Virtually all cells that showed $\mathbf{E}, \mathbf{G}$, and $\mathbf{I}$, NO accumulation were dead as assessed by $\mathbf{F}, \mathbf{H}$, and $\mathbf{J}$, intracellular PI fluorescence. Cells that did not show intracellular PI fluorescence did not show NO accumulation either. Arrows point to rare cells that showed NO accumulation (green fluorescence in original image) but had not yet died (as assessed by membrane permeability to PI and, thus, intracellular red fluorescence in original image). $\mathbf{K}$ and $\mathbf{L}$, No NO accumulation and no programmed cell death were seen in response to virulent bacteria (DC3000 empty vector at $2 \times 10^{7}$ bacteria $\mathrm{ml}^{-1}$ at these timepoints (images taken $5 \mathrm{~h}$ postinoculation). Scale bar $=100 \mu \mathrm{m}$ (all panels at identical level of magnification except inset in $\mathbf{C}$. 
extracellular at this timepoint (see below). In Figure 2D, no PI internalization was seen. The lag between NO accumulation and PI internalization was very short; therefore, this clear difference in staining was not obvious in all replicates of the experiment. Because evidence has been presented that changes to membrane permeability represent a very late stage in cell death (Heath 1998; Heath et al. 1997), the best interpretation of these images is that NO accumulation was first seen close to the time of cell death.

The number of cells in a given field showing DAF-FM triazole fluorescence then increased with time in parallel with the number of cells showing internal PI fluorescence (Fig. 2E to J). In these pictures, most cells that have accumulated NO have undergone PCD as assessed by PI internalization. Arrows point to the rare cells that showed NO accumulation but had not yet died. All dead cells in all images showed NO accumulation.
Neither NO production (Fig. 2K) nor cell death as assessed by PI internalization (Fig. 2L) was seen in response to the isogenic virulent strain carrying the empty plasmid vector at any timepoint examined.

\section{Changes in time of onset and initial extent of hypersensitive cell death are correlated with changes in NO accumulation.}

The closeness in association of timing of NO accumulation with that of PCD was unexpected; therefore, this point was further investigated. HR progression in response to DC3000.avrRpt2 is slower than in response to DC3000.avrB (Grant et al. 2000; Ritter and Dangl 1996; Shapiro and Zhang 2001). As such, if NO accumulation is associated with very late events in hypersensitive death of a cell, kinetics of NO accumulation also should be slower in response to
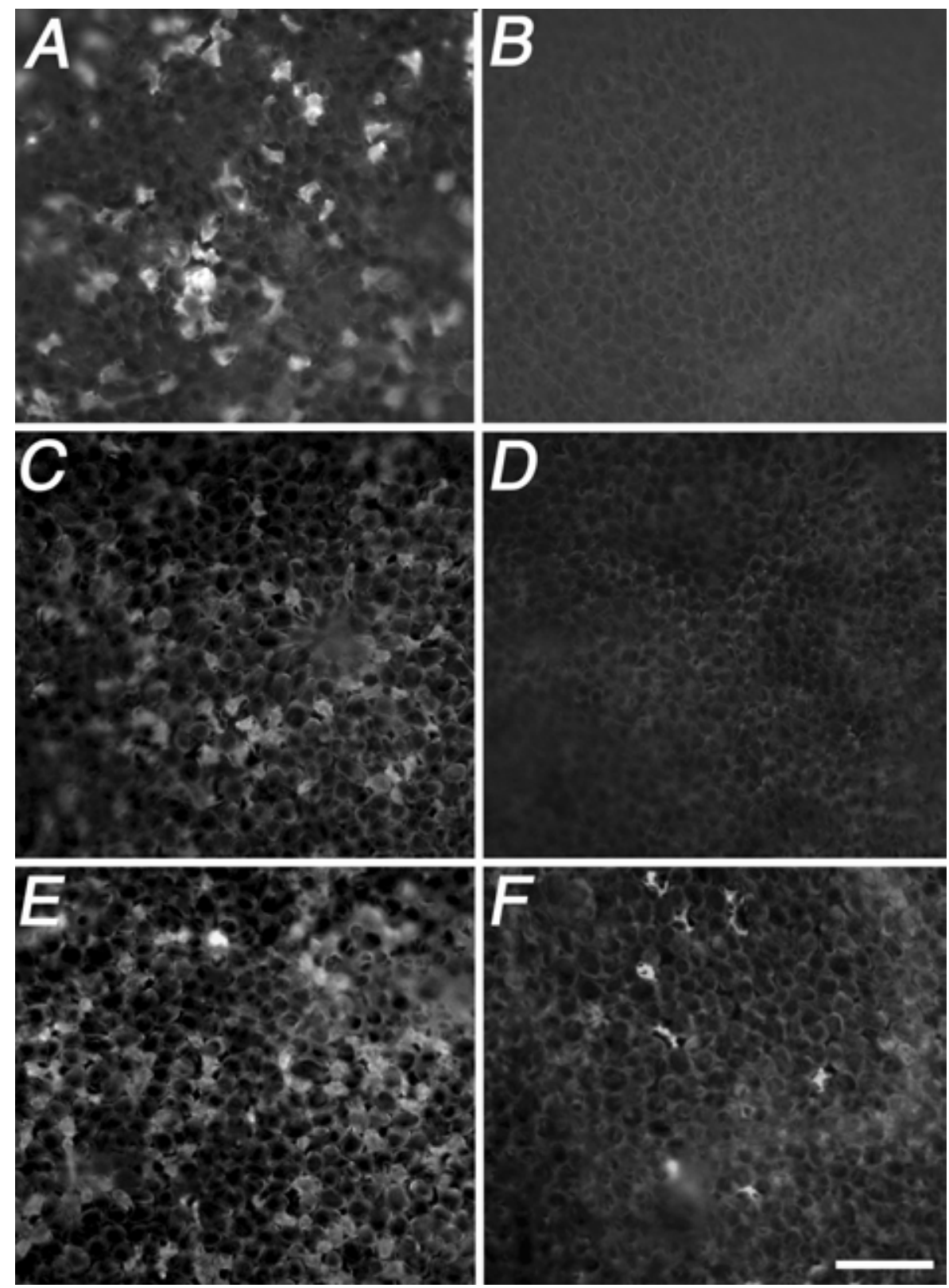

Fig. 3. Kinetics of nitric oxide (NO) accumulation were correlated with kinetics of hypersensitive response progression. A, NO accumulation $7 \mathrm{~h}$ postinoculation of Columbia wild-type plant with DC3000-avrRpt2 at $2 \times 10^{7}$ bacteria ml ${ }^{-1}$. B, No NO accumulation was seen in $n d r 1-1$ mutant plant infected with DC3000.avrRpt2 (picture taken $7 \mathrm{~h}$ postinoculation). C, NO accumulation $4 \mathrm{~h}$ postinoculation of Columbia wild-type plant with DC3000 avrB at $2 \times 10^{7}$ bacteria $\mathrm{ml}^{-1}$. D, NO accumulation $4 \mathrm{~h}$ postinoculation of $n d r 1-1$ mutant plant with DC3000 avrB at $2 \times 10^{7}$ bacteria ml ${ }^{-1}$. E, $\mathrm{NO}^{-}$ accumulation $4.5 \mathrm{~h}$ postinoculation of Columbia wild-type plant with DC3000 $\mathrm{avrB}$ at $2 \times 10^{7}$ bacteria ml $^{-1}$. F, NO accumulation $4.5 \mathrm{~h}$ postinoculation of ndr1-1 mutant plant with DC3000 avrB at $2 \times 10^{7}$ bacteria $\mathrm{ml}^{-1}$. Scale bar $=100 \mu \mathrm{m}$ (all panels at identical level of magnification). 
DC3000-avrRpt2. NO accumulation was first observed in isolated cells $5.5 \mathrm{~h}$ postinoculation of Columbia wild-type plants. Figure 3A, which was taken $7 \mathrm{~h}$ post-inoculation, shows numerous cells that have accumulated NO. The kinetics of PI internalization were similar (data not shown). Mutant ndrl-1 plants do not show a macroscopic HR to DC3000.avrRpt2 under these conditions (Century et al. 1995, 1997). Infection of ndrl-1 plants led to no appreciable accumulation of NO (Fig. 3B) or cell death assessed by PI internalization (data not shown) at any timepoint examined.

We have reported previously that $n d r l-1$ plants infected with DC3000.avrB showed an early, slight delay in HR progression relative to Columbia wild-type plants (Shapiro and Zhang 2001). This data was based on measurement of hydrogen peroxide accumulation, which we showed occurred subsequent to PCD, most likely in cells surrounding the dead cells. Using the PI internalization assay for cell death reproduced this slight delay (data not shown). NO accumulation was delayed to a similar extent in $n d r 1-1$ plants. Parallel experiments using $n d r l-1$ and Columbia plants showed less DAF-FM triazole fluorescence in $n d r l-1$ plants both $4 \mathrm{~h}$ postinoculation (Fig. $3 \mathrm{C}$ and D) and $4.5 \mathrm{~h}$ postinoculation (Fig. $3 \mathrm{E}$ and F). Differences could no longer be seen at later timepoints (data not shown). Thus, timing of NO accumulation was similar to that of PCD in all experiments performed.

\section{Inhibition of NO accumulation leads to a delay in $H R$ progression.}

Next, experiments were undertaken to determine if $\mathrm{NO}$ accumulation was required for PCD. A time course of NO accumulation and cell death (assayed as PI internalization) following infection with DC3000 avrB is shown in Figure 4A. In an experiment done in parallel in which the NOS inhibitor LNMMA was coinfiltrated (Fig. 4B), neither NO accumulation nor cell death was seen 3 h 20 min postinfection. L-NMMA effectively prevented NO accumulation for $4 \mathrm{~h}$. The 4-h timepoint showed one localized region that was beginning to show DAF-FM triazole staining. In most repetitions of this experiment, no cells showed staining (data not shown). However, numerous dead cells were seen in the image shown as assessed by PI internalization. By the 5-h timepoint, L-NMMA inhibition of NO biosynthesis was no longer completely effective, although marked reduction was seen in NO levels (compare with corresponding panel in Fig. 4A). The number of dead cells was not distinguishable from that seen in the absence of L-NMMA. Use of the NO scavenger cPTIO led to similar results (Fig. 4C). No NO accumulation was seen at either the 3-h 20-min timepoint or the 4-h timepoint. By contrast, cell death was seen at the 4-h timepoint. At $5 \mathrm{~h}$ postinoculation, although cPTIO was no longer completely effective at scavenging $\mathrm{NO}$, a marked reduction in NO levels was seen. At this timepoint, the number of dead cells resembled that seen in the absence of cPTIO (compare with Fig 4A).

These reagents delayed rather than blocked cell death. The occurrence of cell death at points when NO could not be detected leads to the conclusion that $\mathrm{NO}$ accumulation cannot be required for cell death. However, NO accumulation did contribute to the rate of $\mathrm{HR}$ progression.

\section{Subcellular and intercellular locations of NO accumulation during HR progression.}

Although epifluorescence microscopy allowed investigation of kinetics of $\mathrm{NO}$ accumulation relative to HR progression, the resolution was not sufficient to determine subcellular localization of the response. Confocal microscopy was used to establish the localization of $\mathrm{NO}$ accumulation and how it changed with time. The timing of cellular events could not be compared directly with those occurring in vivo. With high-resolution confocal microscopy data, the best images were obtained when leaves were placed in water. However, the kinetics of both NO accumulation and HR progression were much slower once a leaf was placed under water. As such, a lower numerical aperture lens (NA 0.75) was used for time courses of confocal imaging of leaves in air. As expected, image quality was reduced when compared with high NA immersion lens imaging (data not shown) because samples were viewed without a coverslip and mounting medium. Nonetheless, these studies verified, under conditions where timing of NO accumulation and HR progression were similar to those observed with epifluorescence microscopy, that the identical order of events was seen as that observed using high-resolution confocal microscopy on leaves placed in water (see below).

In order to determine a time course of changes in cellular localization of NO relative to HR progression, the same fields of cells were observed periodically for several hours. Merged images are presented showing DAF-FM triazole fluorescence (green) and cytoplasmic PI fluorescence (red). PI fluorescence is readily distinguished from chloroplast fluorescence in these images by the characteristic shape of chloroplasts (seen lining cell peripheries) and by the collapsed appearance of most cells showing cytoplasmic PI fluorescence. Differences in emission spectra also were used to confirm distinctions between PI fluorescence and chlorophyll autofluorescence (see Materials and Methods).

The earliest NO accumulation was seen exclusively in the extracellular space. The same field of cells, with the observations spaced $1 \mathrm{~h}$ apart, is shown in Figure 5A and B. Some of the cells adjacent to areas of intercellular fluorescence (marked with arrows) that did not show intracellular NO accumulation at the earlier timepoint (Fig. 5A) did show diffuse intracellular fluorescence at the later timepoint (Fig. $5 \mathrm{~B})$. An area of the same leaf observed later in the response is shown in Figure 5C. Numerous dead cells showing intracellular and extracellular DAF-FM triazole fluorescence were seen. The arrows point to an extracellular region near the dead cells that did not show DAF-FM triazole fluorescence. When the same area was observed at a later timepoint (Fig. 5D), this area did show NO accumulation. We also followed numerous cells that showed extracellular and intracellular DAF-FM triazole fluorescence that subsequently showed PI internalization (data not shown). Repeated observations from replicate experiments established the following order of events. First, extracellular NO accumulated. Then, NO was seen in the cytoplasm of some nearby cells. Many of these cells died soon after. Next, extracellular NO was seen adjacent to these cells and subsequently in distal regions. Then cells in the distal regions displayed cytoplasmic NO accumulation followed by cell death. This pattern was seen to repeat, culminating with the death of most cells in the leaf. This pattern of events is consistent with NO functioning in cell-to-cell spread of the HR.

Higher magnification images of leaves observed $4 \mathrm{~h}$ postinoculation showed the initial sites of NO accumulation as punctate foci located at the cell surface (Fig. 5E). These foci did not move when observed over time. In contrast, chloroplasts initially displayed rapid movement indicative of vigorous cytoplasmic streaming. The lack of movement suggested that these foci were either in the plasma membrane or in the cell wall. The arrow (Fig. 5E) points to a cell that had begun to display intracellular DAF-FM triazole fluorescence. Several punctate foci of fluorescence at the cell surface and a nearby region of extracellular fluorescence remained visible. These punctate foci also were observed in epidermal cells (Fig. 5F). 


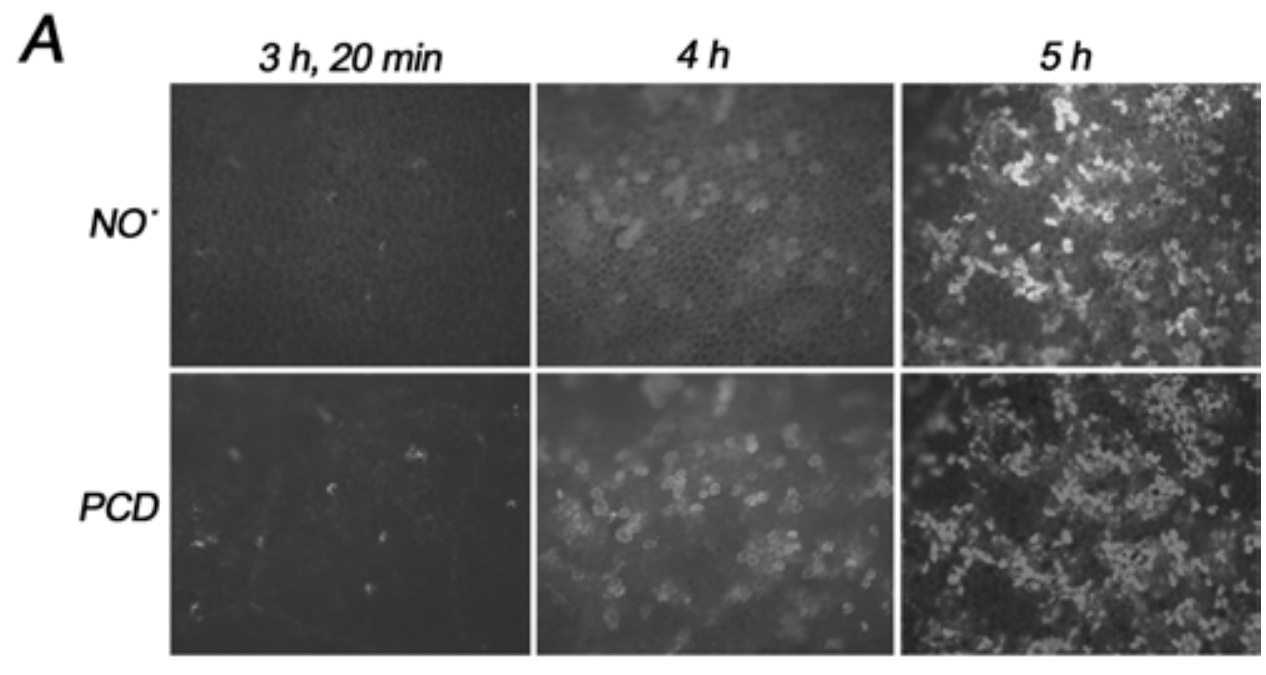

$B$
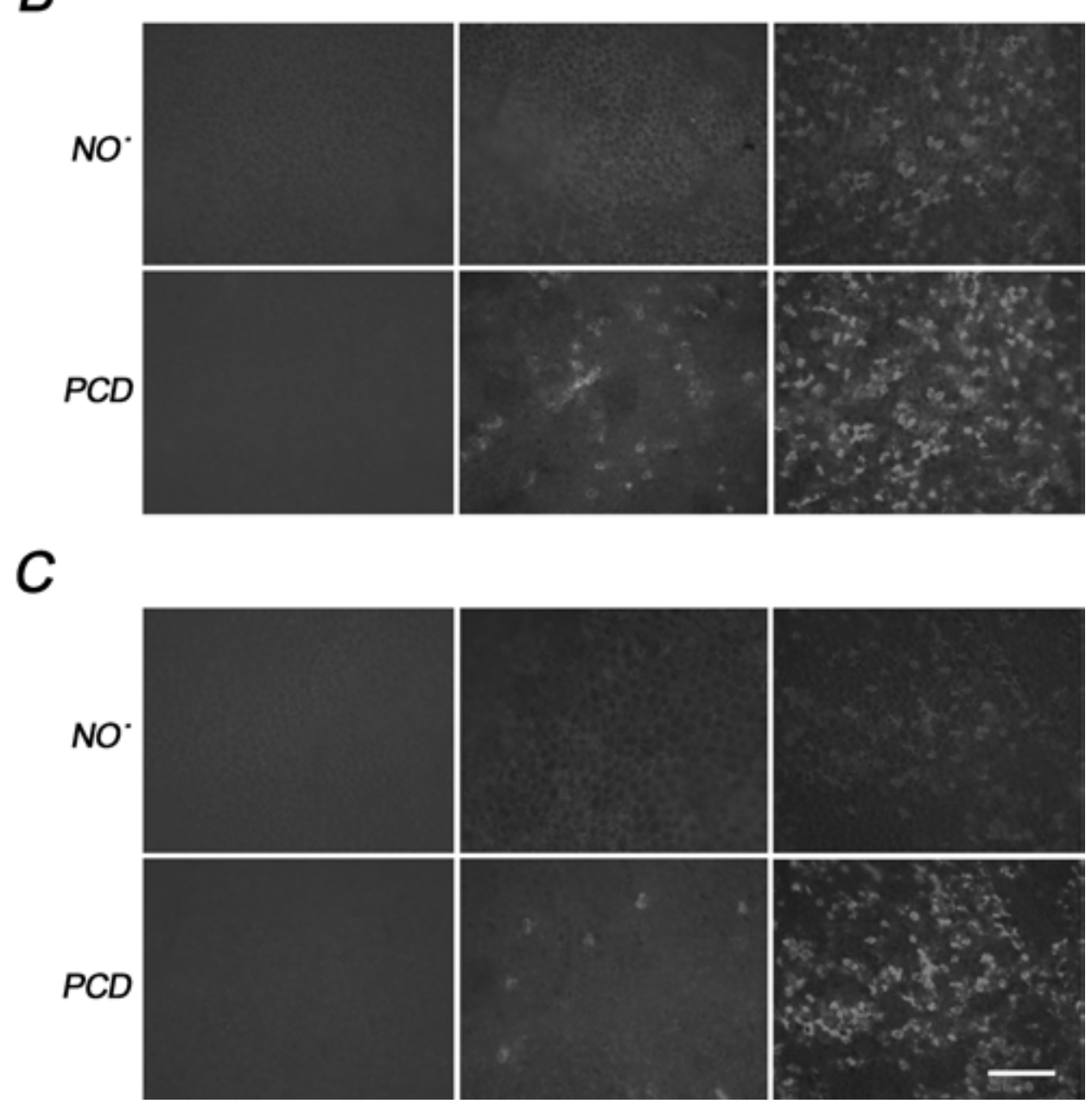

Fig. 4. Nitric oxide (NO) scavenger or a nitric oxide synthase (NOS) inhibitor delayed hypersensitive cell death. All pictures were taken from an experiment performed within a single day and represent typical results. Pairs of images at each indicated timepoint for each treatment were from the same leaf areas. A, NO accumulation and cell death measured as intracellular propidium iodide (PI) fluorescence at the indicated timepoints postinoculation with DC3000 avrB at $2 \times 10^{7}$ bacteria $\mathrm{ml}^{-1}$. B, Inhibition of NO biosynthesis using L-N $\mathrm{N}^{\mathrm{G}}$-monomethyl-arginine monoacetate (L-NMMA) delayed cell death. At $3 \mathrm{~h} 20$ min postinfection, no NO accumulation and no cell death were seen (compare with same timepoints in A). At 4 h postinfection, virtually no NO was seen. The photo shows only one localized area with $\mathrm{NO}$ accumulation. Most trials of this experiment showed no NO accumulation at all at this timepoint. As such, inhibition of NO biosynthesis can be considered fully effective up to this point. Extensive cell death was seen at this timepoint. At later timepoints (e.g., $5 \mathrm{~h}$ ), inhibition of NO biosynthesis was no longer fully effective (although comparison with A shows that it was partially effective). Levels of cell death were indistinguishable at this timepoint from those in the absence of L-NMMA. C, Scavenging of NO using 2-(4-carboxyphenyl)4,4,5,5-tetramethylimidazoline-1-oxyl-3-oxide potassium salt (cPTIO) delayed cell death. At $3 \mathrm{~h} 20$ min postinfection, no NO accumulation and no cell death were seen (compare with same timepoints in A). At $4 \mathrm{~h}$ postinfection, no NO was seen, but cell death was seen. At later timepoints (e.g., 5 h), NO scavenging was no longer fully effective in that low levels of NO accumulation were seen. Levels of cell death were indistinguishable at this timepoint from those in the absence of cPTIO (compare with A). Scale bar $=100 \mu \mathrm{m}$ (all panels at identical level of magnification). 


\section{DISCUSSION}

Based largely on cell culture experiments, models have been proposed in which the central event driving the HR is the accumulation of a precise balance of $\mathrm{NO}$ and reactive oxygen that triggers signal transduction leading to PCD (Delledonne et al. 2002; Hancock et al. 2002; Huang et al. 2002a). The data presented in this article showed that these models do not apply to Arabidopsis leaves. NO accumulation cannot be an early signaling event, because it was not seen until approximately the time of the first PCD events in the HR. Using PI internalization as an assay for cell death, only a slight delay was noted between NO accumulation and PCD. Moreover, it has been noted that changes in plasma membrane permeability are a very late event in hypersensitive cell death (Heath 1998; Heath et al. 1997). In these studies, other markers of cell death (e.g., cytoplasmic condensation) were seen well before changes in plasma membrane permeability. As such, the brief delay seen between onset of DAF-FM triazole fluorescence and PI internalization cannot be taken as
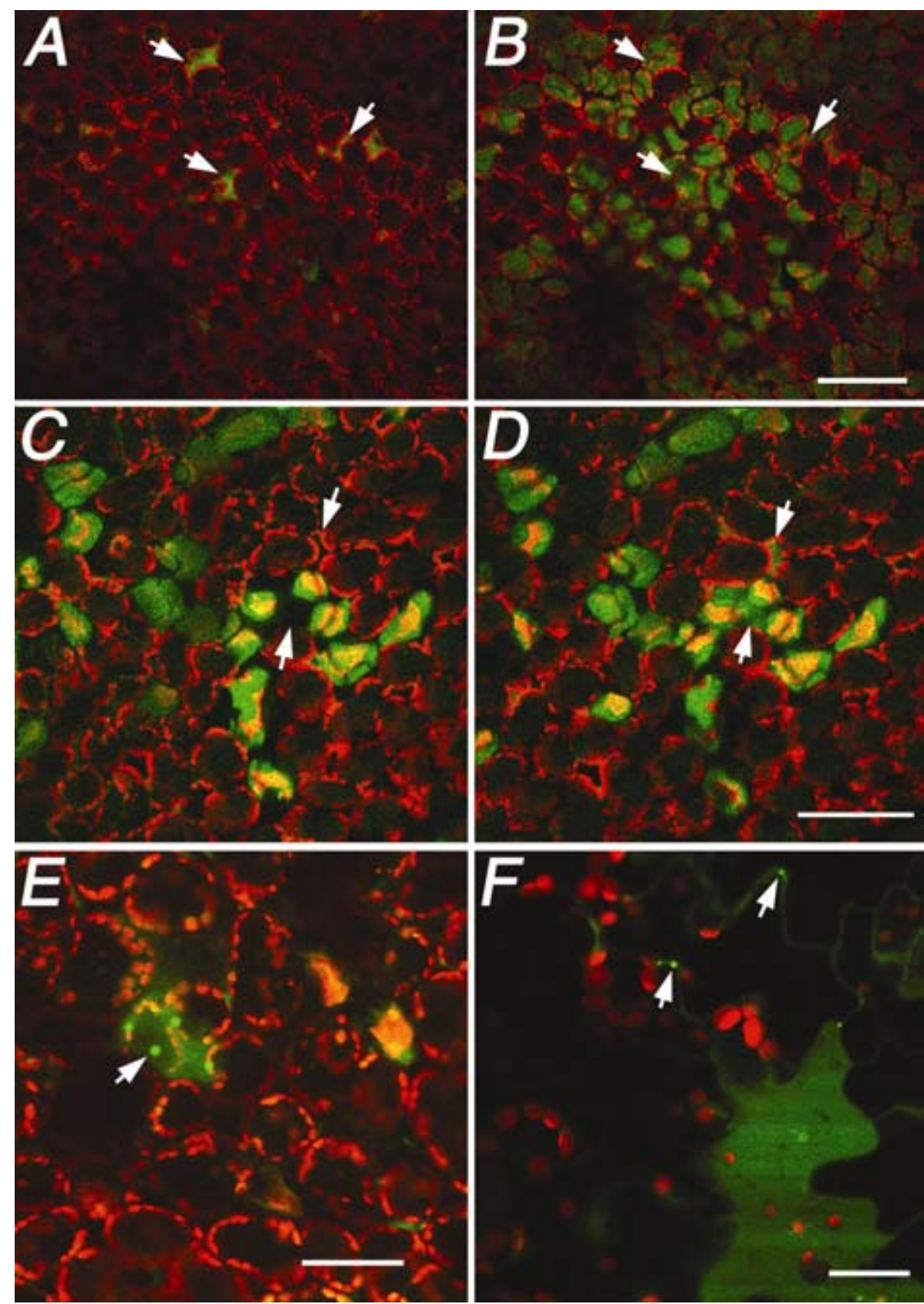

Fig. 5. Changes in nitric oxide (NO) localization with time were consistent with a role in cell-to-cell signaling. All panels show leaves inoculated with DC3000 avrB at $2 \times 10^{7}$ bacteria $\mathrm{ml}^{-1}$. Confocal microscopy was performed on leaves submerged in water. Time points cannot be compared with experiments presented in other figures because hypersensitive response progression was slowed measurably by submergence of leaves in water. A, Photo taken $3 \mathrm{~h} 55 \mathrm{~min}$ postinoculation. B, Same field as in A observed $4 \mathrm{~h} 55 \mathrm{~min}$ postinoculation. Arrows point to regions of intercellular NO accumulation with nearby cells that showed intracellular NO in B but not in A. Scale bar $=100 \mu \mathrm{m}$ (same level of magnification used in A). C, Different area of the same leaf used in A and B. Photo taken $5 \mathrm{~h} 37$ min postinoculation. D, Same field as in C observed $6 \mathrm{~h} 20$ min postinoculation. Arrows point to a region that showed intercellular NO accumulation in D but not in C. Scale bar $=100 \mu \mathrm{m}$ (same level of magnification used in C). $\mathbf{E}$ and $\mathbf{F}$, Higher magnification views show sites of initial $\mathrm{NO}$ accumulation ( $4 \mathrm{~h}$ postinoculation). Green fluorescence was seen as punctate foci at the cell surface (arrows). Cell perimeters are delineated by chloroplast autofluorescence (red, larger punctate spots). Dead cells were visibly collapsed and filled with propidium iodide (red) dye. Scale bars $=50 \mu \mathrm{m}$ in $\mathrm{E}$ and $20 \mu \mathrm{m}$ in F. 
proof of a slight delay between NO accumulation and PCD. Instead, these kinetic experiments established that these two events occurred at approximately the same time. This coincidence of timing was seen in response to two different strains of avirulent bacteria in infections of both wild-type plants and mutant plants with altered timing of the HR.

Exposure of plants to artificially high levels of NO (or reactive oxygen) can lead to PCD. In cell culture experiments, much greater synchrony of response can be achieved (especially in experiments with abiotic elicitors). It is likely that events that would be extremely minor in intact plants (such as early $\mathrm{NO}$ or reactive oxygen production) could be amplified by simultaneous occurrence in numerous cells in close proximity. PCD in response to signaling triggered by oxidative or nitrosative stress is common to diverse eukaryotes. These same mechanisms of triggering PCD might play some role late in HR progression.

By contrast, early events in the HR occur in response to delivery of avr proteins to individual leaf cells. Avr proteins are delivered to Arabidopsis cells within the first $75 \mathrm{~min}$ postinfection of Arabidopsis leaves (Grant et al. 2000). No differences in timing of this event, assessed as timing of loss of efficacy of antibiotics in inhibiting the HR, were noted in that study between avrRpt2, avrB, and avrRpm1. Early signal transduction components would act immediately subsequent to this point. By that criterion, calcium influxes are kinetically competent to be an early component of signaling. Additional evidence also supports this contention (Grant et al. 2000). However, since neither NO accumulation (this study) nor high-level reactive oxygen accumulation (Shapiro and Zhang 2001) occurred at this point, they cannot be major signal transduction elements that trigger the HR, at least in Arabidopsis leaves.

Nonetheless, the experiments with an NO scavenger and an NOS inhibitor demonstrated that NO did play some role in HR progression. However, these reagents were not fully effective in blocking the HR. They merely delayed HR progression by approximately $1 \mathrm{~h}$ (Fig. 4). We also have noted only delays or partial blocks in assays of macroscopic leaf collapse using these reagents (C. K. Worley, C. Zhang, and A. D. Shapiro, unpublished data). The inability of these reagents to block PCD completely has been noted consistently in the literature (Clarke et al. 2000; Delledonne et al. 1998; Foissner et al. 2000; Huang and Knopp 1998). Although these reagents did lose activity with time, likely due to either metabolism or sequestration, results presented in Figure 4 indicated that this couldn't have been the only reason for incomplete blockage of the HR.

At least two possible explanations can be offered for these findings. If NO indeed acts primarily in cell-to-cell signaling, it may not be the only signal. Other signals also may be passed between cells, facilitating HR progression. Alternatively, the partial effects may be due to the nature of the experimental conditions used in studying the HR. HR assays require high titers of pathogen. It is conceivable that cell-tocell signaling is dispensable under these conditions, because sufficient bacteria are inoculated to kill most cells in the plant eventually, even if only cell-autonomous responses occur. Perhaps, with levels of inoculum closer to what would be experienced under field conditions, NO-dependent cell-tocell signaling would play a more important role. At present, neither of these hypotheses can be ruled out. Identification of the cellular targets of NO action that function in the HR will be necessary to resolve this issue.

A function for NO in cell-to-cell signaling would not be unprecedented. Two of the major roles of NO in mammalian biology involve cell-to-cell signaling. NO is the endothelium- derived relaxing factor produced in response to shear stress or endogenous blood pressure regulators such as bradykinin and acetylcholine (Rees et al. 1989). Blood vessel endothelial cells produce NO, which diffuses into nearby smooth muscle cells. NO-induced muscle cell relaxation results in reduced blood pressure. NO also is a retrograde messenger that induces longterm potentiation in the mammalian brain (Arancio et al. 1996). Post-synaptic cells make and release NO. The NO diffuses out of the post-synaptic cell, across the extracellular space, and into the presynaptic cell. NO acts to strengthen the synapse, leading to enhanced responses to subsequent action potentials.

In conclusion, we have demonstrated that NO is not an early signaling intermediate in the Arabidopsis HR. We have demonstrated that neither NO (this study), reactive oxygen (Shapiro and Zhang 2001), nor salicylic acid (Shapiro and Zhang 2001) play essential roles in the first hypersensitive cell death events; therefore, it remains an important challenge for future research to identify components linking molecular recognition of avirulent pathogens to PCD. Nonetheless, impairing NO accumulation can slow down HR progression. NO appears to function in cell-to-cell spread of the HR. This role identifies a common theme in NO signaling between plants and animals, as does the participation of an NOS-like enzyme in NO biosynthesis. It remains to be seen whether NO perception and signaling in target cells also will resemble mammalian paradigms or will be unique to plants.

\section{MATERIALS AND METHODS}

\section{Plants, bacteria, and inoculations.}

The Arabidopsis Columbia and ndrl-1 lines were grown and Pseudomonas strains were cultured and used as described (Century et al. 1995; Shapiro and Zhang 2001; Zhang and Shapiro 2002). Arabidopsis double mutant nia1-1/nia2-5 seed (Col-0 background) were obtained from the Arabidopsis Biological Resource Center (stock number CS2356) and grown on soil supplemented with $2 \mathrm{mM}\left(\mathrm{NH}_{4}\right)_{2} \mathrm{SO}_{4}$. Bacteria was used at $2 \times 10^{7} \mathrm{ml}^{-1}$ (optical density at $600 \mathrm{~nm}=0.02$ ), and inoculations were performed as previously described (Shapiro 2000).

\section{Chemicals.}

DAF-FM diacetate and PI were purchased from Molecular Probes (Eugene, OR). L-NMMA was purchased from Toronto Research Chemicals (Toronto, Canada). cPTIO and SNP were purchased from Sigma-Aldrich (St. Louis). L-NMMA and cPTIO were dissolved in water as $10 \times$ stocks and used at final concentrations of $1 \mathrm{mM}$ and $100 \mu \mathrm{M}$, respectively.

\section{Measuring NO accumulation.}

DAF-FM diacetate was dissolved in dimethyl sulfoxide to make a 5-mM stock solution that was diluted 400-fold in water immediately prior to use. Leaves were infiltrated with the DAF-FM diacetate solution 15 min prior to harvest through the same needle hole originally used to inoculate with bacteria. Images were taken using an Axioskop 2 upright microscope (Carl Zeiss MicroImaging, Inc., Thornwood, NY, U.S.A.) equipped with a $1.6 \mathrm{M}^{2} \mathrm{BIO}$ lens and an Axiocam digital camera. Leaves were excised and immediately put directly under the lens for imaging. Green fluorescence was excited using a 100-W mercury arc lamp with a GFP470 excitation filter and detected using a GFP525 emission filter. Constant exposure time was used for all experiments. For every experiment shown, at least three independent replicates gave consistent results. 


\section{PI internalization assay for cell death.}

PI was dissolved in deionized water to make a stock $(1 \mathrm{mg}$ $\mathrm{ml}^{-1}$ ) that was diluted 1,000-fold prior to use. Inoculation of plants with bacteria and subsequent dye infiltration were precisely as with NO quantitation. For experiments using both dyes, one solution containing both was introduced. Imaging was as with NO measurements, except that PI was imaged using a rhodamine filter cube (540/25 BP excitation and $605 / 55$ BP emission filters). Focal planes with mostly epidermal cells yielded results comparable to those with mostly mesophyll cells in all experiments with this assay and with NO quantitation.

\section{Confocal microscopy.}

Leaves were inoculated with bacteria and subsequently infiltrated with DAF-FM diacetate and PI as described above. Leaves were excised and immediately put into a Lab-Tek II chambered cover glass (Fischer Scientific, Pittsburgh) and submerged in deionized water. Double-labeled DAF-FM triazole and propidium iodide confocal images were acquired on a Zeiss LSM 510 NLO equipped Axiovert 200M using either a Zeiss 40X Plan-Neofluar oil immersion (NA 1.3) or Zeiss 20X Plan-Apochromat (NA 0.75) objective lens. In order to eliminate bleed through, images were collected in fastline switch mode using the 488-nm laser line of a $25-\mathrm{mW}$ Argon laser (LASOS, Jena, Germany) with appropriate 500-550 BP emission filter (DAF-FM diacetate) and the 543-nm laser line of a 1-mW Helium:Neon laser (LASOS) with appropriate 560 LP emission filter (PI). All data acquisition settings (laser power, pinhole size, filters, detector settings, scan conditions, and so on) were identical for all treatments. Extracellular localization of fluorescence was confirmed through focus imaging of multiple planes. All images were edited using Photoshop 6.0 (Adobe, San Jose, CA, U.S.A.).

\section{ACKNOWLEDGMENTS}

This research was supported by a Young Professor Award from DuPont Corporation to A. D. Shapiro. C. Zhang was supported in part by a predoctoral research assistantship from the University of Delaware College of Agriculture and Natural Resources and by a University Competitive Fellowship. Published as paper \#1743 in the Journal Series of the Delaware Agricultural Experiment Station.

\section{LITERATURE CITED}

Arancio, O., Kiebler, M., Lee, C. J., Lev-Ram, V., Tsien, R. Y., Kandel, E. R., and Hawkins, R. D. 1996. Nitric oxide acts directly in the presynaptic neuron to produce long-term potentiation in cultured hippocampal neurons. Cell 87:1025-1035.

Arndtjovin, D. J., and Jovin, T. M. 1989. Fluorescence labeling and microscopy of DNA. Pages 417-448 in: Methods in Cell Biology. Vol. 30. Academic Press, Inc. San Diego, CA, U.S.A.

Barroso, J. B., Corpas, F. J., Carreras, A., Sandalio, L. M., Valderrama, R., Palma, J. M., Lupíánez, J. A., and del Rio, L. A. 1999. Localization of nitric oxide synthase in plant peroxisomes. J. Biol. Chem. 274:36729-36733.

Beligni, M. V., Fath, A., Bethke, P. C., Lamattina, L., and Jones, R. L. 2002. Nitric oxide acts as an antioxidant and delays programmed cell death in barley aleurone layers. Plant Physiol. 129:1642-1650.

Beligni, M. V., and Lamattina, L. 1999. Nitric oxide counteracts cytotoxic processes mediated by reactive oxygen species in plant tissues. Planta 208:337-344.

Century, K. S., Holub, E. B., and Staskawicz, B. J. 1995. NDR1, a locus of Arabidopsis thaliana that is required for disease resistance to both a bacterial and a fungal pathogen. Proc. Natl. Acad. Sci. U.S.A. 92:6597-6601.

Century, K. S., Shapiro, A. D., Repetti, P. P., Dahlbeck, D., Holub, E., and Staskawicz, B. J. 1997. NDR1: A pathogen-induced component required for Arabidopsis disease resistance. Science 278:1963-1965.

Chandok, M. R., Ytterberg, A. J., van Wljk, K. J., and Klessig, D. F. 2003. The pathogen-inducible nitric oxide synthase (iNOS) in plants is a variant of the $\mathrm{P}$ protein of the glycine decarboxylase complex. Cell 113:469-482.

Clarke, A., Desikan, R., Hurst, R. D., Hancock, J. T., and Neill, S. J. 2000. NO way back: Nitric oxide and programmed cell death in Arabidopsis thaliana suspension cultures. Plant J. 24:667-677.

Delledonne, M., Murgia, I., Ederle, D., Sbicego, P. F., Biondani, A., Polverari, A., and Lamb, C. 2002. Reactive oxygen intermediates modulate nitric oxide signaling in the plant hypersensitive response. Plant Physiol. Biochem. 40:605-610.

Delledonne, M., Xia, Y., Dixon, R. A., and Lamb, C. 1998. Nitric oxide functions as a signal in plant disease resistance. Nature 394:585-588.

Delledonne, M., Zeier, J., Marocco, A., and Lamb, C. 2001. Signal interactions between nitric oxide and reactive oxygen intermediates in the plant hypersensitive disease resistance response. Proc. Natl. Acad. Sci. U.S.A. 98:13454-13459.

de Pinto, M. C., Tommasi, F., and de Gara, L. 2002. Changes in the antioxidant systems as part of the signaling pathway responsible for the programmed cell death activated by nitric oxide and reactive oxygen species in Bright-Yellow 2 cells. Plant Physiol. 130:698-708.

Desikan, R., Griffiths, R., Hancock, J., and Neill, S. 2002. A new role for an old enzyme: Nitrate reductase-mediated nitric oxide generation is required for abscisic acid-induced stomatal closure in Arabidopsis thaliana. Proc. Natl. Acad. Sci. U.S.A. 99:16314-16318.

Durner, J., Wendehenne, D., and Klessig, D. F. 1998. Defense gene induction in tobacco by nitric oxide, cyclic GMP, and cyclic ADPribose. Proc. Natl. Acad. Sci. U.S.A. 95:10328-10333.

Foissner, I., Wendehenne, D., Langebartels, C., and Durner, J. 2000. In vivo imaging of an elicitor-induced nitric oxide burst in tobacco. Plant J. 23:817-824.

Garcês, H., Durzan, D., and Pedroso, M. C. 2001. Mechanical stress elicits nitric oxide formation and DNA fragmentation in Arabidopsis thaliana. Ann. Bot. 87:567-574.

Goodman, R. N., and Novacky, A. J. 1994. The Hypersensitive Response in Plants to Pathogens: A Resistance Phenomenon. American Phytopathological Society. St. Paul, MN.

Grant, M., Brown, I., Adams, S., Knight, M., Ainslie, A., and Mansfield, J. 2000. The RPM1 plant disease resistance gene facilitates a rapid and sustained increase in cytosolic calcium that is necessary for the oxidative burst and hypersensitive cell death. Plant J. 23:441-450.

Hancock, J. T., Desikan, R., Clarke, A., Hurst, R. D., and Neill, S. J. 2002. Cell signaling following plant/pathogen interactions involves the generation of reactive oxygen and nitrogen species. Plant Physiol. Biochem. 40:611-617.

Heath, M. C. 1998. Involvement of reactive oxygen species in the response of resistant (hypersensitive) or susceptible cowpeas to the cowpea rust fungus. New Phytol. 138:251-263.

Heath, M. C., Nimchuck, Z. L., and Xu, H. 1997. Plant nuclear migrations as indicators of critical interactions between resistant or susceptible cowpea epidermal cells and invasion hyphae of the cowpea rust fungus. New Phytol. 135:689-700.

Hu, X. Y., Neill, S. J., Cai, W. M., and Tang, Z. C. 2003. NO-mediated hypersensitive responses of rice suspension cultures induced by an incompatible elicitor. Chin. Sci. Bull. 48:358-363.

Huang, J., and Knopp, J. A. 1998. Involvement of nitric oxide in Ralstonia solanacearum-induced hypersensitive reaction in tobacco. Pages 218-224 in: Bacterial Wilt Disease: Molecular and Ecological Aspects. P. Prior, C. Allen, and J. Elphinstone, eds. INRA and Springer Editions, Berlin.

Huang, X., Kiefer, E., von Rad, U., Ernst, D., Foissner, I., and Durner, J. 2002a. Nitric oxide burst and nitric oxide-dependent gene induction in plants. Plant Physiol. Biochem. 40:625-631.

Huang, X., von Rad, U., and Durner, J. 2002b. Nitric oxide induces transcriptional activation of the nitric oxide-tolerant alternative oxidase in Arabidopsis suspension cells. Planta 215:914-923.

Itoh, Y., Ma, F. H., Hoshi, H., Oka, M., Noda, K., Ukai, Y., Kojima, H., Nagano, T., and Toda, N. 2000. Determination and bioimaging method for nitric oxide in biological specimens by diaminofluorescein fluorometry. Anal Biochem. 287:203-209.

Kojima, H., Urano, Y., Kikuchi, K., Higuchi, T., Hirata, Y., and Nagano, T. 1999. Fluorescent indicators for imaging nitric oxide production. Angew Chem. Int. Ed. Engl. 38:3209-3212.

Koyama, H., Toda, T., and Hara, T. 2001. Brief exposure to low-pH stress causes irreversible damage to the growing root in Arabidopsis thaliana: Pectin-Ca interaction may play an important role in proton rhizotoxicity. J. Exp. Bot. 52:361-368.

Pedroso, M. C., Magalhaes, J. R., and Durzan, D. 2000a. A nitric oxide burst precedes apoptosis in Angiosperm and Gymnosperm callus cells and foliar tissues. J. Exp. Bot. 51:1027-1036.

Pedroso, M. C., Magalhaes, J. R., and Durzan, D. 2000b. Nitric oxide induces cell death in Taxus cells. Plant Sci. 157:173-180. 
Rees, D. D., Palmer, R. M. J., and Moncada, S. 1989. Role of endothelium-derived nitric oxide in the regulation of blood pressure. Proc. Natl. Acad. Sci. U.S.A. 86:3375-3378.

Ribeiro, E. A. J., Cuhna, F. Q., Tamashiro, W. M. S. C., and Martins, I. S. 1999. Growth phase-dependent subcellular localization of nitric oxide synthase in maize cells. FEBS (Fed. Eur. Biochem. Soc.) Lett. 445:283-286.

Ritter, C., and Dangl, J. L. 1996. Interference between two specific pathogen recognition events mediated by distinct plant disease resistance genes. Plant Cell 8:251-257.

Shapiro, A. D. 2000. Using Arabidopsis mutants to delineate disease resistance signaling pathways. Can. J. Plant Pathol. 22:199-216.

Shapiro, A. D., and Zhang, C. 2001. The role of NDR1 in avirulence gene-directed signaling and control of programmed cell death in Arabidopsis. Plant Physiol. 127:1089-1101.

Wilkinson, J. Q., and Crawford, N. M. 1993. Identification and characterization of a chlorate-resistant mutant of Arabidopsis thaliana with mutations in both nitrate reductase structural genes NIA1 and NIA2. Mol. Gen. Genet. 239:289-297.

Zhang, C., and Shapiro, A. D. 2002. Two pathways act in an additive rather than obligatorily synergistic fashion to induce systemic acquired resistance and $P R$ gene expression. BMC Plant Biol. 2:9.

Zhang, C., Gutsche, A. T., and Shapiro, A. D. Submitted for publication.

Zottini, M., Formentin, E., Scattolin, M., Carimi, F., Lo Schiavo, F., and Terzi, M. 2002. Nitric oxide affects plant mitochondrial functionality in vivo. FEBS (Fed. Euro. Biochem. Soc.) Lett. 515:75-78. 